Copyright (C) 2014 IEEE. Personal use of this material is permitted. Permission from IEEE must be obtained for all other uses, in any current or future media, including reprinting/republishing this material for advertising or promotional purposes, creating new collective works, for resale or redistribution to servers or lists, or reuse of any copyrighted component of this work in other works. 


\title{
Feedback Cancellation with Probe Shaping Compensation
}

\author{
*C. Renato C. Nakagawa, Student Member, IEEE, Sven Nordholm, Senior Member, IEEE, Wei-Yong Yan
}

\begin{abstract}
Adaptive feedback cancellation methods may integrate the use of probe signals to assist with the biased optimal solution in acoustic systems working in closed-loop. However, injecting a probe noise in the loudspeaker decreases the signal quality perceived by users of assistive listening devices. To counter this, probe signals are usually shaped to provide some level of perceptual masking. In this letter we show the impact of using a shaping filter on the system behavior in terms of convergence rate and steady state error. From this study, it can be concluded that shaping the probe signal may have detrimental influence in terms of system performance. Accordingly, we propose to use the unshaped probe signal combined with an inverse filter of the shaping filter to identify the feedback channel. This restructure of the problem restores convergence rate of LMS type algorithms. Furthermore, we also show that an adequate forward path delay is required to obtain an unbiased solution and that the suggested scheme reduces this delay.
\end{abstract}

Index Terms-Acoustic feedback, bias problem, feedback cancellation, hearing aids, probe injection

\section{INTRODUCTION}

Acoustic feedback occurs when part of the loudspeaker signal from an audio system is picked up by its microphone creating an acoustic loop. The signal traveling around this loop gets re-amplified for each round trip potentially causing audible artifacts, such as howling. Systems susceptible to feedback problems include public address systems and assistive listening devices. The feedback limits the maximum stable gain achievable, it deteriorates the sound quality by producing a distortion of the incoming signal, and it is a cause of instability in acoustic systems working in closed-loop [1].

The use of adaptive filters as feedback cancelers is a common method to compensate for the feedback signal. However, one of the main challenges with feedback cancelers is the well known bias problem where the filter's estimates become biased when there is correlation between the loudspeaker and incoming signal [1] [3]. This correlation generally leads to a poor system performance and in the worst-case scenario, it causes the cancellation system to fail. Different techniques have been proposed to reduce this correlation including phase modification, frequency shifting, nonlinear processing, decorrelating pre-filters, probe noise injection, and the use of multiple microphones to estimate the incoming signal and remove it prior to adapting the canceler [1], [2], [4]-[6].

In this letter we analyze a particular method of using an injected probe signal as the input to the canceler, where the canceler bases the estimation of the feedback path on the probe signal. The work in [7] presented new insights into the bias problem when a probe signal is used as input to the canceler. The feedback canceler's optimum solution is biased [3], even if the probe signal is white noise. To obtain an unbiased solution, [7] suggested the use of an adequate delay in the forward path. However, if the probe signal is spectrally shaped, then the optimal solution may still be biased. The work in [2] notes that "shaping the probe signal decreases the decorrelation effect, making the noise injection less effective in removing the bias", nevertheless, [2] does not present any further details on this issue.

Copyright (c) 2014 IEEE. Personal use of this material is permitted. However, permission to use this material for any other purposes must be obtained from the IEEE by sending a request to pubs-permissions@iee.org.

The authors are with the Dept. of Electr. \& Comput. Eng., Curtin Univ., Bentley, WA, Australia (e-mail: carlos.nakagawa@postgrad.curtin.edu.au; s.nordholm@curtin.edu.au; w.yan@exchange.curtin.edu.au).
Thus, there is a need to gain more insights on system performance as a result of shaping the probe signal. Understanding this is important for making further improvements.

To study the impact on the canceler's performance resulting from shaping the probe signal, we use the methodology presented in [8] and show the influence a shaping filter has on system behavior. The work in [8], [9] presents the notion of a frequency domain measure, called the power transfer function (PTF), which is used to approximately predict system behavior such as convergence rate and steady-state error. Then, we extend the delay condition from [7] to obtain an unbiased solution. Finally, we present a new approach which employes a filter that compensates for the shaping filter. This improves system performance while maintaining the benefits which arise from perceptually shaping the probe signal.

The proposed approach results in a similar structure to that of the prediction error method (PEM) presented in [5] where pre-filters are applied to pre-whiten the signals. It may also seem similar to the work in [10] where pre-filters are used as enhancement filters to increase the probe to disturbing (incoming) signal ratio. The purpose of the pre-filters in [5] is to decorrelate the incoming and loudspeaker signals to reduce the bias in the canceler's solution. However, the aim of the proposed approach is to remove the negative impact of the shaping filter on system behavior. Furthermore, an adequate forward path delay is sufficient to decorrelated the signals with the proposed method. In [10] the aim is to reduce the disturbing signal power without changing the probe noise power by adapting long-term prediction error filters which results in improved system performance. However, in this work, the purpose of our equalizing filter is to compensate for the use of a shaping filter which also leads to improved system performance.

This paper is organized as follows. In Sec. II a system description for acoustic feedback cancellation using a shaped probe signal is given. In Sec. III the delay condition from [7] is extended to take into account the probe signal correlation. Following this, Sec. IV shows how the shaping filter impacts system behavior. Then, in Sec. V a new approach is presented that deals with the detrimental influence from shaping the probe signal. In Sec. VI simulation results are rendered to validate the analysis presented. Finally, Sec. VII concludes the paper.

\section{SYSTEM DESCRIPTION}

Figure 1 illustrates a feedback canceler for an assistive listening device with a single microphone. The feedback path between the loudspeaker and the microphone is assumed to be a discrete-time finite impulse response (FIR) filter with coefficient vector $\mathbf{g}=$ $\left[\begin{array}{llll}g_{0} & g_{1} & \ldots & g_{L_{g}-1}\end{array}\right]^{T}$ with filter length $L_{g}$ which is represented as a polynomial transfer function $G(q)$ in $q$ as $G(q)=\mathbf{g}^{T} \mathbf{q}$ with $\mathbf{q}=\left[\begin{array}{llll}1 & q^{-1} & \ldots & q^{-L_{g}+1}\end{array}\right]^{T}$. This representation allows the following notation, for the filtering of $y(n)$ by $G(q), G(q) y(n)=$ $\mathbf{g}^{T} \mathbf{y}(n)$ [11]. Column vectors are emphasized using lower letters in bold, the superscript $\mathrm{T}$ denotes vector transpose, the discrete-time index is denoted by $n$, and the symbol $q^{-1}$ denotes the discrete-time delay operator $q^{-1} u(n)=u(n-1)$. All signals are real-valued, and we denote all signals as discrete-time signals with time index $n$ for convenience.

The forward path $K(q)$ represents the regular signal processing path of the device. In this work, $K(q)$ has a delay $d_{k} \geqslant$ 


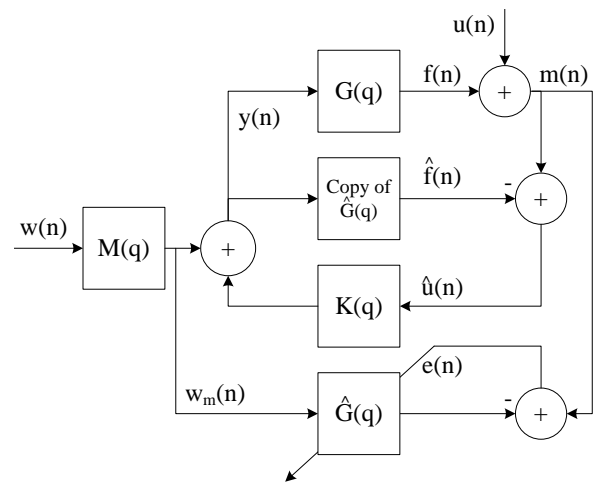

Figure 1. Traditional injected probe signal approach for acoustic feedback cancellation. With shaped probe signal used as input to the canceler.

1 and provides the system with a constant gain i.e., $K(q)=$ $q^{-d_{k}} K$. The adaptive filter $\hat{G}(q)$, with coefficient vector $\hat{\mathrm{g}}=$ $\left[\begin{array}{llll}\hat{g}_{0} & \hat{g}_{1} & \ldots & \hat{g}_{L_{g}-1}\end{array}\right]^{T}$, identifies and tracks changes to the feedback path, where we assume sufficient order with $L_{\hat{g}}=L_{g}$. If $L_{\hat{g}}<L_{g}$ then the system is undermodelled and the canceler's solution will be biased.

A shaped probe signal $w_{m}(n)$ is used as the input to the feedback canceler $\hat{G}(q)$ and injected into the loudspeaker signal $y(n)$. The probe signal $w_{m}(n)$ is generated as $w_{m}(n)=M(q) w(n)$, where $w(n)$ is a white noise sequence, and $M(q)$ is a known spectral shaping filter which is designed to provide some kind of perceptual masking of the noise signal.

The loudspeaker signal is defined as

$$
\begin{aligned}
y(n)= & K \cdot S(q) u\left(n-d_{k}\right)+w_{m}(n) \\
& +K \cdot(G(q)-\hat{G}(q)) S(q) w_{m}\left(n-d_{k}\right)
\end{aligned}
$$

where $u(n)$ is the incoming signal which, we assume in our analysis, is a zero-mean stationary stochastic signal with correlation function $r_{u}(k)=E\{u(n) u(n-k)\}, E\{\cdot\}$ denotes the expectation operator, and the sensitivity function

$$
S(q)=\frac{1}{1-K(q)(G(q)-\hat{G}(q))} .
$$

The frequency function $K(\omega)(G(\omega)-\hat{G}(\omega))$ in (2) is often referred to as the "loop-response", where the frequency response for $K(q)$ and $G(q)$ is denoted by $K(\omega)$ and $G(\omega)$, respectively, with $\omega=[0,2 \pi]$. The Nyquist criterion states that oscillations may occur if the magnitude response of the loop-gain is greater than unity and the loop-phase is a multiple of $2 \pi$ [12]. It can be seen in (2) that the path $G(q)$ may lead to system instability. To avoid this, the amount of gain $K(q)$ has to be limited. However, if the feedback canceler $\hat{G}(q)$ can resemble $G(q)$, then the system is brought closer to its desired response and $S(q)=1$.

The microphone signal is then given as

$$
\begin{aligned}
m(n)= & u(n)+G(q) y(n) \\
= & u(n)+K \cdot G(q) S(q) u\left(n-d_{k}\right)+G(q) w_{m}(n) \\
& +K \cdot G(q)(G(q)-\hat{G}(q)) S(q) w_{m}\left(n-d_{k}\right) .
\end{aligned}
$$

From Fig. 1, the error signal is defined as

$$
\begin{aligned}
e(n)= & m(n)-\hat{G}(q) w_{m}(n) \\
= & u(n)+K \cdot G(q) S(q) u\left(n-d_{k}\right) \\
& +(G(q)-\hat{G}(q)) w_{m}(n) \\
& +K \cdot G(q)(G(q)-\hat{G}(q)) S(q) w_{m}\left(n-d_{k}\right) .
\end{aligned}
$$

As presented in [7], the optimal solution in the mean square sense is biased even if $w_{m}(n)$ is white noise as a result of the last term in (4), where it is assumed that $w_{m}(n)$ and $u(n)$ are uncorrelated. However, [7] showed that if an adequate forward path delay is in place $d_{k} \geqslant L_{g}$ then an unbiased solution is obtained when $w_{m}(n)$ is white noise, i.e., in the absence of $M(q)$. Next we study the impact of shaping the probe signal on the feedback cancellation system.

\section{DELAY CONDITION FOR UNBIASED SOLUTION}

First, we look at the condition required for an unbiased estimate. From Section II, it was presented that if the probe signal is shaped, then a bias term may arise. In such a case, the forward path delay has to be sufficiently long so that the correlation introduced by the shaping filter does not contribute to a bias term. We assume that the shaped probe signal $w_{m}(n)$ will have a finite correlation function, i.e. $r_{w_{m}}(k)=0 \forall|k|>k_{w_{m}}$, where $k_{w_{m}}$ is a finite integer number. If we also take into account the delay condition presented in [7], then the delay condition for an unbiased solution is given by

$$
d_{k} \geqslant L_{g}+k_{w_{m}} \text {. }
$$

Note that if we assume that $K(q)=q^{-d_{k}} \bar{K}(q)$, a more general forward path with $L_{\bar{k}}$ the length of $\bar{K}(q)$, then $\bar{K}(q)$ will add correlation to the solution. Thus, the delay condition needs to include $L_{\bar{k}}$, i.e., in this more general case the delay condition is $d_{k} \geqslant L_{g}+k_{w_{m}}+L_{\bar{k}}$.

\section{PROBE SHAPING IMPACT ON SYSTEM BEHAVIOR}

We are interested in studying the impact that shaping the probe signal has on the system's convergence rate, steady-state behavior, and tracking error. To accomplish this, we follow the methodology presented in [9] where the notion of an estimate of a PTF measure is presented and used to give insights into the system's performance. The work in [9] uses open-loop signals and the closed-loop effects are ignored, nevertheless, it provides a reasonable estimate for system behavior in closed-loop without requiring knowledge of the feedback path.

In [9] the estimate of the PTF is defined as $\hat{\xi}(\Omega, n) \approx$ $E\left\{\tilde{G}(\Omega, n) \tilde{G}^{*}(\Omega, n)\right\}$ where the feedback path is assumed to be time-varying, $\tilde{G}(n)=E\left\{\tilde{\mathbf{g}}(n) \tilde{\mathbf{g}}^{T}(n)\right\}, \tilde{\mathbf{g}}(n)=\hat{\mathbf{g}}(n)-\mathbf{g}(n)$, and $\Omega$ is the discrete frequency bin. The estimate $\hat{\xi}(\Omega, n)$, the diagonal elements of the DFT of $\tilde{G}(n)$ (assumed to be a Toeplitz matrix), for a single microphone setup can be written as

$$
\begin{aligned}
\hat{\xi}(\Omega, n)= & \left(1-2 \mu(n) S_{w_{m}}(\Omega)\right) \hat{\xi}(\Omega, n-1)+L_{g} \mu^{2}(n) \\
& \cdot\left(S_{w_{m}}(\Omega) S_{u}(\Omega)\right)+S_{\check{g}}(\Omega)
\end{aligned}
$$

where $S_{w_{m}}(\Omega)$ denotes the power spectrum density (PSD) of the shaped probe noise signal $w_{m}(n), S_{u}(\Omega)$ denotes the auto PSD of the incoming signal $u(n)$, and $S_{\check{g}}(\Omega)$ is the covariance of the feedback path changes. Eq. (6) was derived for the least-mean square (LMS) algorithm under the assumptions of sufficiently small step size $\mu(n)$ and large model order parameter $L_{g}$. It is also assumed that $\tilde{G}(n)$ is a Toepliz matrix. However, this assumption is valid if the feedback path is assumed to be a stationary stochastic variable.

The PTF approximate expression can be viewed as a first-order difference equation in $\hat{\xi}(\Omega, n)$ described by the transfer function $Z(q)=\frac{\beta}{1-\alpha q^{-1}}$. The coefficient $\alpha$ determines the pole location in $Z(q)$ and thus the decay rate of $\hat{\xi}(\Omega, n)$ [8]. The decay rate of the $\mathrm{PTF}$ for a single microphone is given as

$$
\alpha=1-2 \mu(n)|M(\Omega)|^{2} S_{w}(\Omega)
$$

and convergence rate $(\mathrm{CR})$ in $\mathrm{dB} /$ iteration is thus given by $\mathrm{CR}=$ $10 \log _{10}(|\alpha|)$, where $S_{w}(\Omega)$ is the PSD for $w(n)$, and $M(\Omega)$ is 


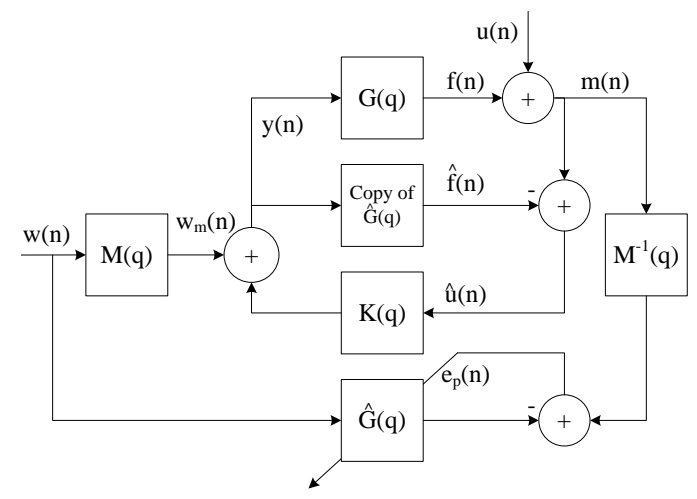

Figure 2. Proposed method. Probe signal $w(n)$ is used as the input into the canceler $\hat{G}(q)$ instead of $w_{m}(n)$. Also, $m(n)$ is filtered by $M^{-1}(q)$ prior to calculating $e_{p}(n)$.

the frequency response for $M(q)$. The steady-state (SS) behavior, $\hat{\xi}(\Omega, n)=\lim _{n \rightarrow \infty} \hat{\xi}(\Omega, n)$, is presented as

$$
\mathrm{SS}=\underbrace{\lim _{n \rightarrow \infty} L_{g} \frac{\mu(n)}{2} S_{u}(\Omega)}_{\text {Steady-state error }}+\underbrace{\lim _{n \rightarrow \infty} \frac{S_{\check{g}}(\Omega)}{2 \mu(n)|M(\Omega)|^{2} S_{w}(\Omega)}}_{\text {Tracking error }} .
$$

Using (7) and (8) it can be seen that the shaping filter $M(q)$ impacts the system behavior, more specifically, the convergence rate and tracking error of the system. For instance, a probe signal may be shaped to have a long-term average speech spectrum as seen in simulations in [5]. Considering this scenario we may intuitively interpret (7) and (8). From (7) it can be observed that at higher frequencies, the frequencies of interest in a feedback cancellation problem, the convergence rate will be slower and tracking error higher as a result of a small $|M(\Omega)|^{2}$. At the same time, lower frequencies will carry a higher weight which may lead to an unstable system. Thus, a very small step size may be required to achieve convergence depending on the level of the incoming signal. Therefore, it can be seen that the shaping filter may negatively impact system behavior.

\section{PRobe Shaping COMPENSATION}

From seeing the impact of shaping the probe signal on system performance, we now propose a method that improves system performance. This method, presented in Fig. 2, can be viewed as an extension of the traditional probe driven system according to Fig. 1. The main observations to be made from Fig. 2 is that the noise probe signal $w(n)$ is used as the input into the adaptive algorithm instead of $w_{m}(n)$. Also, the definition of the error signal is modified, where the microphone signal is filtered by $M^{-1}(q)$ prior to calculating $e_{p}(n)$. Thus, $M(q)$ is designed so that its inverse $M^{-1}(q)$ exists. Note that the shaped probe signal $w_{m}(n)$ is still injected to the loudspeaker signal with the aim to render the injected noise less perceptual. The new error signal $e_{p}(n)$ is defined as

$$
\begin{aligned}
e_{p}(n)= & M^{-1}(q) m(n)-\hat{G}(q) w(n) \\
= & M^{-1}(q)\left(u(n)+K \cdot G(q) S(q) u\left(n-d_{k}\right)\right) \\
& +(G(q)-\hat{G}(q)) w(n) \\
& +K \cdot G(q)(G(q)-\hat{G}(q)) S(q) w\left(n-d_{k}\right) .
\end{aligned}
$$

The convergence rate and the tracking error for the proposed approach will now be both independent of $|M(\Omega)|^{2}$ from (7) and (8). However, $\left|M^{-1}(\Omega)\right|^{2}$ will now influence the steady-state error. The convergence rate for the proposed approach can be approximated by $\mathrm{CR}_{p}=10 \log _{10}\left(\left|\alpha_{p}\right|\right)$ in $\mathrm{dB} /$ iteration where

$$
\alpha_{p}=1-2 \mu(n) S_{w}(\Omega)
$$
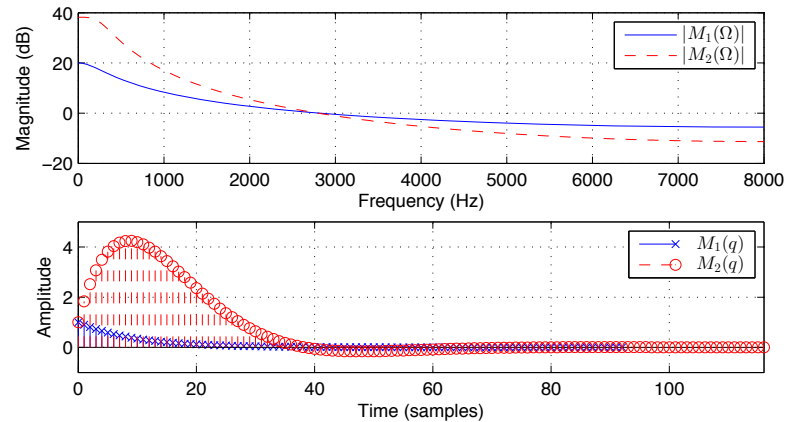

Figure 3. Frequency and impulse responses for $M_{1}(q)$ and $M_{2}(q)$.

and the steady-state behavior is presented as

$$
\mathrm{SS}_{p}=\lim _{n \rightarrow \infty} L_{g} \frac{\mu(n)}{2} \frac{S_{u}(\Omega)}{|M(\Omega)|^{2}}+\lim _{n \rightarrow \infty} \frac{S_{\check{g}}(\Omega)}{2 \mu(n) S_{w}(\Omega)} .
$$

By comparing (7) and (8) with (10) and (11) a trade-off between convergence rate and steady-state error can be seen. That is, the cost for higher convergence rate is a higher value for the steady-state error. In the particular case where the incoming signal is considered to be speech signals, we can expect that at lower frequencies, where the disturbance (incoming signal) is most dominant, the convergence rate will be slower with a much lower steady-state value. At higher frequencies, frequencies of interest, the convergence rate will be higher with a small degradation in steady-state performance. This is presented and verified in more details in Section VI.

\section{A. Delay condition for proposed approach}

Another benefit with the proposed approach is that it reduces the forward path delay $d_{k}$ required to decorrelate the closed-loop signals to produce an unbiased solution, especially if $k_{w_{m}}$ is large. This is most beneficial with open fitted assistive listening devices where the forward path delay must be sufficiently small. Thus, the condition on the forward path delay with the proposed approach is

$$
d_{k} \geqslant L_{g}
$$

and is no longer dependent on $k_{w_{m}}$. It can be shown that with an adequate forward path delay (12), and assuming that $w(n)$ is uncorrelated with $M^{-1}(q) u(n)$, an unbiased solution is still obtainable.

\section{B. Comment on more general $M(q)$}

It must be pointed out that $M(q)$ may not always have an inverse, especially if $M(q)$ is designed based on statistical information of $u(n), w(n)$, and some masking threshold to perceptually mask the noise. The design of $M(q)$ is beyond the scope of this work. Nevertheless, in the case where $M(q)$ is not invertible, we wish to design a filter which estimates an equalizer for $M(q)$. One potential solution is to carry out a least-squares fit between the known signals $w_{m}(n)$ and $w(n)$ to obtain the coefficients for a compensation filter. Then, both the microphone signal and the input signal into the canceler are filtered prior to adaptation.

\section{Simulation RESUlts}

In this section we verify some of the theoretical analysis presented. Let $M(q)$ shape the probe signal with a long-term average speech spectrum which can be modeled with a low-order, autoregressive (AR) random process. Two fixed, invertible models, were used to shape the probe signal. A first order model $M_{1}(q)$, as used in [13] to generate a sequence with a long term speechlike spectrum, is defined as $M_{1}(q)=\left(1-0.9 q^{-1}\right)^{-1}$ and a 
Table I

PTF ESTIMATE, CONVERGENCE RATE (DB/ITERATION).

\begin{tabular}{|c||c|c|c|c|}
\hline$\Omega$ & $1(500 \mathrm{~Hz})$ & $3(1.5 \mathrm{kHz})$ & $5(2.5 \mathrm{kHz})$ & $7(3.5 \mathrm{kHz})$ \\
\hline \hline$M_{1}(q)$ & $-1.74 \mathrm{e}-02$ & $-3.90 \mathrm{e}-03$ & $-1.18 \mathrm{e}-03$ & $-5.54 \mathrm{e}-04$ \\
\hline$M_{2}(q)$ & $-1.35 \mathrm{e}+00$ & $-1.23 \mathrm{e}-01$ & $-8.73 \mathrm{e}-03$ & $-1.80 \mathrm{e}-03$ \\
\hline Prop. & $-1.74 \mathrm{e}-04$ & $-1.74 \mathrm{e}-04$ & $-1.74 \mathrm{e}-04$ & $-1.74 \mathrm{e}-04$ \\
\hline
\end{tabular} \begin{tabular}{|c|c|c|c|}
\hline $9(4.5 \mathrm{kHz})$ & $11(5.5 \mathrm{kHz})$ & $13(6.5 \mathrm{kHz})$ & $15(7.5 \mathrm{kHz})$ \\
\hline \hline$-3.23 \mathrm{e}-04$ & $-2.14 \mathrm{e}-04$ & $-1.55 \mathrm{e}-04$ & $-1.19 \mathrm{e}-04$ \\
\hline$-5.97 \mathrm{e}-04$ & $-2.59 \mathrm{e}-04$ & $-1.35 \mathrm{e}-04$ & $-7.91 \mathrm{e}-05$ \\
\hline$-1.74 \mathrm{e}-04$ & $-1.74 \mathrm{e}-04$ & $-1.74 \mathrm{e}-04$ & $-1.74 \mathrm{e}-04$ \\
\hline
\end{tabular}

Table II

PTF ESTIMATE, STEADY STATE ERROR (DB).

\begin{tabular}{|c||c|c|c|c|}
\hline$\Omega$ & $1(500 \mathrm{~Hz})$ & $3(1.5 \mathrm{kHz})$ & $5(2.5 \mathrm{kHz})$ & $7(3.5 \mathrm{kHz})$ \\
\hline \hline Traditional & -32.67 & -32.70 & -32.79 & -32.94 \\
\hline Prop. $M_{1}^{-1}(q)$ & -52.67 & -46.21 & -41.11 & -37.98 \\
\hline Prop. $M_{2}^{-1}(q)$ & -70.90 & -61.12 & -49.80 & -43.09 \\
\hline \multicolumn{1}{|c|}{$9(4.5 \mathrm{kHz})$} & $11(5.5 \mathrm{kHz})$ & $13(6.5 \mathrm{kHz})$ & $15(7.5 \mathrm{kHz})$ \\
\hline \hline-33.15 & -33.42 & -33.74 & -34.13 \\
\hline-35.85 & -34.33 & -33.25 & -32.49 \\
\hline-38.51 & -35.16 & -32.63 & -30.72 \\
\hline
\end{tabular}

second order model $M_{2}(q)$, based on [4], is presented as $M_{2}(q)=$ $\left(1-2 \times 0.92 \cos \left(\frac{200 \times 2 \times \pi}{15750}\right) q^{-1}+0.92^{2} q^{-2}\right)^{-1}$.

Figure 3 presents the frequency and impulse response for $M_{1}(q)$ and $M_{2}(q)$. From observing the plots, some of the system behavior may be deduced. We obtain $k_{w_{m}}$ for the delay condition in (5) from the impulse response, where a forward path delay of around 20 or 60 samples, in addition to $L_{g}$, for $M_{1}(q)$ or $M_{2}(q)$ respectively may be required to obtain an unbiased solution.

From the frequency response in Fig. 3 it can be inferred, using (7), that for frequencies over $3 \mathrm{kHz}$ the convergence rate will be relatively slower when the shaping filters $M_{1}(q)$ or $M_{2}(q)$ are used. And at lower frequencies, where an incoming speech would be most dominant, the convergence rate will be higher (especially with $M_{2}(q)$ ), potentially resulting in system instability. To quantify this, we present the PTF estimate for convergence rate and steady-state error in Tables I and II respectively. The LMS algorithm with the following parameters were used, step size $\mu=0.00002, L_{g}=32$, $h_{u}=\left[\begin{array}{cc}1 & 0.3\end{array}\right]^{T}$ shapes the incoming signal which is a white Gaussian noise (WGN) sequence with unit variance, and $M(q)$ shapes the probe signal which is also a WGN with unit variance.

The convergence rate for the odd numbered frequency bins for the traditional case with $M_{1}(q)$ and $M_{2}(q)$, and the proposed case (same values for both shaping filters) are presented in Table I, where the convergence rate is higher at lower frequencies and slower at higher ones as a result of shaping the probe signal, refer to (7). It can also be seen that the proposed approach restores the convergence rate achieved as if WGN is used instead of the shaped noise, refer to (10). The estimated steady-state values achievable using (8) and (11) are presented in Table II where the probe shaping filter $M(q)$ does not affect the steady-state values for the traditional approach, however, it comes into effect with the proposed approach based on (11).

Next, to validate the estimates from Tables I and II, we present in Fig. 4 three sub-figures (low, mid, and high frequencies) comparing the traditional probe shaped approach with the proposed showing the estimated and true PTF curves. For the simulations, to obtain the true PTF curves, the LMS algorithm is used in closed-loop, with step size $\mu=0.00002$, and a forward path gain of $K=0 \mathrm{~dB}$. The incoming signal is a WGN sequence with unit variance and shaped by $h_{u}=\left[\begin{array}{ll}1 & 0.3\end{array}\right]^{T}$, and the probe signal is also WGN

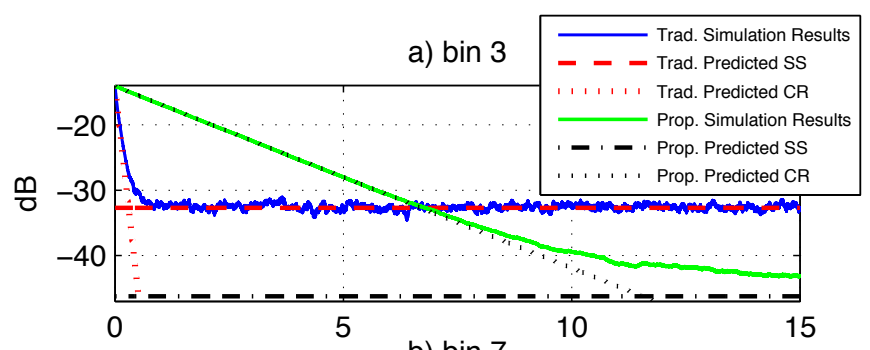

b) bin 7
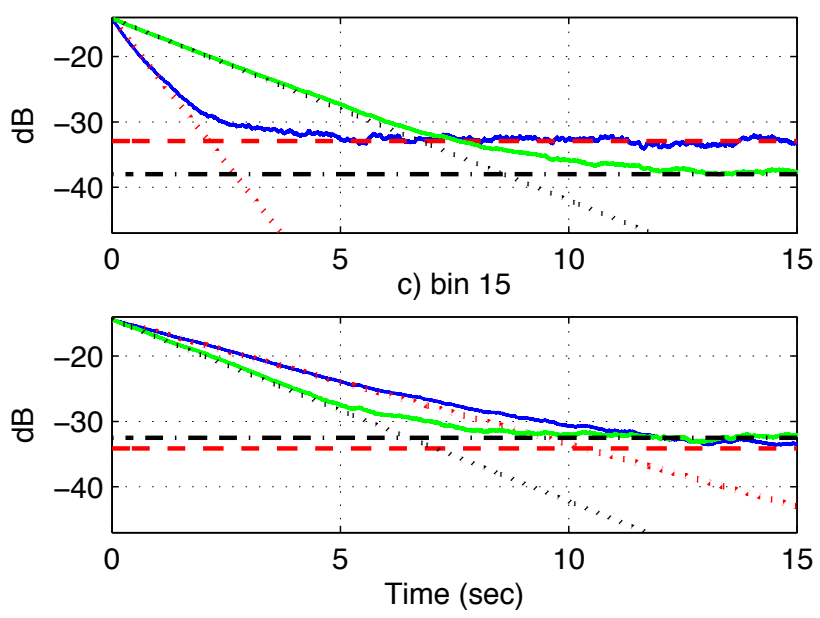

Figure 4. Plots presenting estimated and true PTF curves for three frequency bins: bin $3(1.5 \mathrm{kHz})$, bin $7(3.5 \mathrm{kHz})$, and bin $15(7.5 \mathrm{kHz})$. Where $M_{1}(q)$ was used as the shaping filter.

with unit variance but filtered by $M_{1}(q)$. A forward path delay of $d_{k}=64$ samples $(4 \mathrm{~ms})$ was used and $L_{\hat{g}}=L_{g}=32$ samples. The feedback is considered to be a random Gaussian channel with variance $\sigma_{g}^{2}=0.001$. In each simulation run, new realizations of Gaussian stochastic sequences are drawn. Figure 4 presents the true PTF averaged values for 100 simulation runs.

From the plots in Fig. 4 the influence of the probe shaping filter can be seen. It is observed that the convergence rate for the traditional approach is faster at lower frequencies than those at higher ones, and the steady-state values are not affected by $M_{1}(q)$. With the proposed approach, the convergence rate is recovered to that as if in the absence of $M_{1}(q)$, and is constant at all frequency bins, which agrees with (10). The trade-off between convergence rate and steady-state error can also be seen. For instance, from observing the plot for bin 3, it can be seen that with the proposed approach, slower convergence rates is achieved while obtaining a lower steady-state error when compared to the traditional approach, whereas faster convergence rates is obtainable at higher frequencies (bin 15) at the cost of slightly higher steady-state error.

\section{CONCLUSION}

Feedback cancellation systems which employ the use of probe signal injection may introduce a shaping filter to perceptually mask the probe signal. This letter studied the impact on system behavior as a result of using a shaping filter. It was found that the shaping filter change the adaptation speed and may also introduce bias in the solution. To combat those limitations, we have proposed a scheme which restores convergence speed. The suggested method uses the unshaped probe signal combined with a filtered version of the microphone signal to identify the feedback channel. By employing these signals in the identification, the adaptive canceler has a restored convergence. Furthermore, we have showed that an adequate forward path delay is sufficient to obtain an unbiased solution and also that the proposed scheme reduces this delay. 


\section{REFERENCES}

[1] A. Spriet, S. Doclo, and M. Moonen, "Feedback control in hearing aids," Springer Handbook of Speech Process., pp. 979-1000, 2008.

[2] T. van Waterschoot and M. Moonen, "Fifty years of acoustic feedback control: state of the art and future challenges," Proc. IEEE, no. 99, pp. $1-40,2011$.

[3] U. Forssell and L. Ljung, "Closed-loop identification revisited," Automatica, vol. 35, no. 7, pp. 1215-1241, Jul. 1999.

[4] J. Hellgren, "Analysis of feedback cancellation in hearing aids with Filtered-x LMS and the direct method of closed loop identification," IEEE Trans. Speech Audio Process., vol. 10, no. 2, pp. 119-131, 2002.

[5] A. Spriet, I. Proudler, M. Moonen, and J. Wouters, "Adaptive feedback cancellation in hearing aids with linear prediction of the desired signal," IEEE Trans. Signal Process., vol. 53, no. 10, pp. 3749-3763, 2005.

[6] C. R. C. Nakagawa, S. Nordholm, and W.-Y. Yan, "Dual Microphone Solution for Acoustic Feedback Cancellation for Assistive Listening," in Proc. IEEE Int. Conf. Acoust., Speech, Signal Process., Mar. 2012, pp. $149-152$.

[7] — "New Insights Into Optimal Acoustic Feedback Cancellation," Signal Process. Lett., IEEE, vol. 20, no. 9, pp. 869-872, 2013.

[8] M. Guo, T. B. Elmedyb, S. H. Jensen, and J. Jensen, "Analysis of Acoustic Feedback/Echo Cancellation in Multiple-Microphone and Single-Loudspeaker Systems Using a Power Transfer Function Method," IEEE Trans. Signal Process., vol. 59, no. 12, pp. 5774-5788, Dec. 2011.

[9] —_, "On Acoustic Feedback Cancellation Using Probe Noise in Multiple-Microphone and Single-Loudspeaker Systems," IEEE Signal Process. Lett., vol. 19, no. 5, pp. 283-286, May 2012.

[10] M. Guo, S. H. Jensen, and J. Jensen, "Novel Acoustic Feedback Cancellation Approaches in Hearing Aid Applications Using Probe Noise and Probe Noise Enhancement," IEEE Trans. Audio. Speech. Lang. Processing, vol. 20, no. 9, pp. 2549-2563, Nov. 2012.

[11] L. Ljung, System Identification: Theory for the User, 2nd ed., 2nd ed. Prentice-Hall, Aug. 1999.

[12] H. Nyquist, "Regeneration Theory," Bell Syst. Tech. J., vol. 11, no. 1, pp. 126-147, 1932.

[13] A. Spriet, K. Eneman, M. Moonen, and J. Wouters, "Objective measures for real-time evaluation of adaptive feedback cancellation algorithms in hearing aids," in Proc. 16th Eur. Signal Process. Conf., 2008. 